\title{
Frecuencia de periodontitis apical en tratamientos endodónticos de pregrado
}

\author{
Frequency of apical periodontitis in endodontic treatment in undergraduate
}

\author{
León $\mathrm{P}^{1}$, Ilabaca MJ1 , Alcota $\mathrm{M}^{2}$, González FE ${ }^{3}$
}

\begin{abstract}
RESUMEN
La periodontitis apical es una enfermedad de los tejidos periapicales de etiología bacteriana. Su tratamiento requiere erradicar los microorganismos del conducto radicular y obturarlo para lograr la reparación posterior. El objetivo de éste trabajo fue determinar la frecuencia de periodontitis apical en la población que asiste a la clínica de Endodoncia de la Facultad de Odontología de la Universidad de Chile y su relación con diferentes variables: diagnóstico específico, edad, sexo, agudas vs. crónicas, éxito/fracaso del tratamiento y rehabilitación post-tratamiento. Se recolectó información de 292 dientes de pacientes tratados en dicha clínica, analizándose la información de aquellos con dientes diagnosticados con algún tipo de periodontitis apical clasificándolo según la nueva nomenclatura de la asociación de endodoncia americana. La frecuencia de periodontitis apical fue de un $36.7 \%$ (92 pacientes), de los cuales un $77.8 \%$ presentaron patologías de tipo crónicas. Un $75 \%$ de los pacientes pertenecieron al sexo femenino y la $5^{\text {ta }}$ década representó un $34.8 \%$ de los pacientes. El $100 \%$ de los pacientes que acudieron a control presentó éxito en sus tratamientos según variables clínico-radiográficas, mientras que la restauración más frecuente post-tratamiento fue la resina compuesta (52.4\%). Dado el pequeño número de pacientes que concurrieron a la citación de control para determinar el éxito del tratamiento, esta variable debe ser considerada como preliminar. Nuestros resultados mostraron que la condición mas frecuente fue la periodontitis apical de tipo crónica, afectando más a mujeres y a la $5^{\text {ta }}$ década. Rev. Clin. Periodoncia Implantol. Rehabil. Oral Vol. 4(3); 126-129, 2011.
\end{abstract}

Palabras clave: Frecuencia de periodontitis apical, tratamiento endodóntico, porcentaje de éxito.

\section{ABSTRACT}

Apical periodontitis is a microbially induced inflammatory disease of the periapical tissues. Its treatment requires eliminating microorganism from root canal and sealing it properly to induce further tissue repair. The aim of this study was to determine the frequency of apical periodontitis and its distribution by gender, age, range, acute vs. chronic, treatment success and rehabilitation in the population attended at the undergraduate clinic of Endodontic at the Faculty of Dentistry, Universidad de Chile. Information was collected from 292 teeth of patients treated at the clinic, recording data of those with teeth diagnosed with some type of apical periodontitis and related to different variables: specific diagnosis, age, gender, acute vs. chronic, successful or failed treatment and post-treatment restoration. The frequency of apical periodontitis was $36.7 \%$ (92 patients). $77.8 \%$ of the teeth with apical periodontitis had a chronic disease. $75 \%$ of the patients were female. The $5^{\text {th }}$ decade represented a $34.8 \%$. There was a $100 \%$ success rate in their treatments, while the most common restoration was the composite $(52.4 \%)$. The low number of patients who responded to the dental revision to determine the success of the treatment, despite the $100 \%$ rate, cannot be evaluated as a significant result. Our data showed that the most frequent condition was chronic apical periodontitis, affecting more female patients, and the $5^{\text {th }}$ decade and incisors.

Rev. Clin. Periodoncia Implantol. Rehabil. Oral Vol. 4(3); 126-129, 2011.

Key words: Frequency of apical periodontitis, success rate, endodontic treatment.

\section{INTRODUCCIÓN}

La Periodontitis Apical (PA) se define como una enfermedad inflamatoria de los tejidos perirradiculares causada por una infección microbiana persistente en el sistema de canales radiculares del diente afectado $^{(1)}$. La interacción dinámica que ocurre en el periápice entre las bacterias patógenas y los mecanismos de defensa del hospedero, trae como resultado varias categorías de periodontitis apicales, las cuales se clasifican en base a sus hallazgos clínicos, radiográficos e histológicos ${ }^{(2)}$. Las lesiones asociadas con síntomas importantes, como dolor o inflamación, corresponden a las agudas o sintomáticas, mientras que aquellas que tienen síntomas ligeros o no los tienen corresponden a las crónicas o asintomáticas ${ }^{(1,3)}$.

El mayor reto que presenta el tratamiento de este tipo de patologías es erradicar o reducir sustancialmente la carga microbiana presente en los conductos radiculares mediante una adecuada preparación quimiomecánica y la obturación de dichos conductos permitiendo tanto la reparación de los tejidos como la recuperación de su funcionalidad ${ }^{(3-6)}$. Se considera que ha existido reparación, y por tanto éxito de los dientes tratados endodónticamente, cuando existe ausencia de sintomatología clínica asociado a una evidente disminución o ausencia total de la radiolucidez inicial del periápice en los controles radiográficos posteriores ${ }^{(7-10)}$. Existen diversos estudios que registran el éxito de tratamiento con porcentajes que van desde un $62 \%$ a un $95 \%{ }^{(7,11-13)}$. En relación a la periodontitis apical, Friedman \& Mor (2004) determinaron un éxito de tratamiento para piezas con dicha patología de un $74 \%$ a un $86 \%$, mientras que para piezas tratadas sin la patología indicaron un rango de éxito de entre un $92 \%$ y un $98 \%{ }^{(14)}$.

Para evaluar el éxito de los tratamientos odontológicos dentro de una comunidad, es importante establecer también la frecuencia de periodontitis apical en dicha población. Estudios epidemiológicos llevados a cabo en diferentes poblaciones han mostrado una alta frecuencia de periodontitis apical, con valores que van desde un $26 \%$ a un $80 \%$, indicando que hasta $2 / 3$ de algunas comunidades necesitan de una terapia endodóntica puntualmente dirigida a la periodontitis

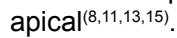

En Chile no se registran estudios de frecuencia ni de evaluación de éxito de tratamiento en dientes con periodontitis apical. El

1. Cirujano Dentista. Universidad de Chile. Chile.

2. Cirujano Dentista. Especialista en Endodoncia, Magíster en Educación en Ciencias de la Salud. Departamento de Odontología Conservadora, Facultad de Odontología, Universidad de Chile. Chile.

3. Cirujano Dentista. Doctor en Ciencias Biomédicas. Departamento de Odontología Conservadora, Facultad de Odontología, Universidad de Chile. Chile.

Correspondencia autores: Fermín E. González, fegonzalez@med.uchile.cl, y Marcela Alcota Rojas, malcota@u.uchile.cl. Departamento de Odontología Conservadora, Facultad de Odontología, Universidad de Chile. Sergio Livingstone 943, 8380453. Independencia, Santiago. Chile. Trabajo recibido el 07/09/2011. Aprobado para su publicación el 21/11/2011. 
objetivo de este estudio fue conocer la frecuencia de periodontitis apical y su distribución en cuanto a su condición de agudas o crónicas, sexo y edad. Adicionalmente, se determinó, de manera preliminar, el éxito de los tratamientos realizados en dientes con esta patología en los pacientes que acudieron a la clínica de pregrado de Endodoncia de la Facultad de Odontología de la Universidad de Chile. Lo anterior permitirá evaluar las necesidades de tratamiento de nuestra población, así como también identificar, revisar y, eventualmente, modificar determinados protocolos de tratamiento aplicados actualmente en la clínica de endodoncia.

\section{MATERIALES Y MÉTODOS}

Se utilizaron 292 fichas de dientes (correspondientes a 254 pacientes) con requerimiento de terapia endodóntica en el área de pregrado de Endodoncia de la Facultad de Odontología de la Universidad de Chile en el año 2009. El protocolo fue aprobado por el comité de ética de la Facultad de Odontología de la Universidad de Chile, y cada paciente que participó en el estudio firmó un consentimiento informado.

En dichas fichas, la patología dentaria fue diagnosticada con exámenes clínico-radiográficos y test de sensibilidad siendo todos los tratamientos efectuados por estudiantes bajo supervisión docente de académicos especialistas en la disciplina. Se citó telefónicamente para un control clínico y radiográfico a todos los pacientes con tratamientos endodónticos efectuados en dientes con el diagnóstico de periodontitis apical, cuyo tiempo post-operatorio fuese de 6 meses o mas. Acudieron al control 21 pacientes ( 21 dientes en total) de 92 posibles (99 dientes en total) los que fueron sometidos a un examen clínico y radiográfico del diente tratado (realizado por un mismo operador) y se registró en una ficha especialmente confeccionada para esta investigación, el estado pre-operatorio del diente según su ficha original y el estado actual (presencia de fístula, absceso, dolor espontáneo, dolor a la percusión y alteraciones de la región vestibular). Se consignó además el tipo de restauración que actualmente presentaba el diente (prótesis fija, amalgama, resina compuesta o cemento temporal) así como el estado en que esta restauración se encontraba (buen o mal estado). Por último, se le realizó una radiografía periapical y se comparó con la radiografía previa al tratamiento.

\section{Criterios de Inclusión y Exclusión}

Para este estudio se consideraron los diagnósticos y nomenclatura propuestos por la Asociación de Endodoncia Americana ${ }^{(16,17)}$ : Periodontitis Apical Asintomática (PAA), Periodontitis Apical Sintomática (PAS), Absceso Apical Agudo (AAA) y Absceso Apical Crónico (AAC). El resto de las indicaciones para terapia endodóntica como patologías pulpares en dientes vitales, indicaciones por rehabilitación o retratamientos por problemas en la obturación no se consideraron. Además, se registraron datos sobre la edad, sexo y diente involucrado.

Para evaluar el éxito del tratamiento, se utilizaron los siguientes criterios:

Éxito: Clínicamente el diente está asintomático y radiográficamente presenta una estructura ósea periapical normal o lesión con evidencias de disminución del tamaño de la lesión en al menos un $50 \%$ luego de 6 meses o más de realizado el tratamiento al compararlas con la radiografía inicial.

Fracaso: Clínicamente presencia de dolor espontáneo o a la percusión, presencia de fístula o absceso, y radiográficamente mantención o aumento del tamaño de la lesión preexistente luego de 6 meses o más de realizado el tratamiento.

\section{RESULTADOS}

De las 292 fichas de dientes revisadas (correspondientes a 254 pacientes) que requerían terapia endodóntica, un total de 92 pacientes (36.7\%) fueron diagnosticados con algún tipo de periodontitis apical. La Tabla 1 muestra la frecuencia de los diferentes diagnósticos.

La Tabla 2 compara la frecuencia de patologías agudas vs. crónicas según número de dientes afectados. Se encontró una mayor frecuencia de las patologías crónicas $(77.8 \%)$, siendo PAA la patología más frecuente con un $54.6 \%$. Entre las patologías agudas, que constituyeron el $22.2 \%$ del total, el AAA fue la patología más frecuente $(17.1 \%)$.
La Tabla 3 muestra la distribución por edades y sexo para pacientes con periodontitis apicales. Encontramos una mayor frecuencia en mujeres $(75 \%)$ que en hombres. En relación a las edades, el grupo con mayor frecuencia corresponde al de 40 a 49 años (34.8\%), mientras los extremos de $10-19$ años $y \geq 60$, presentan la menor frecuencia $(9.8 \%$ y $8.6 \%$ respectivamente).

Para evaluar el éxito de tratamiento en dientes con periodontitis apical, fueron controlados 21 pacientes, el $100 \%$ de los cuales presentaron tratamientos exitosos (Tabla 4 y Figura 1). La Tabla 5 muestra el tipo de rehabilitación posterior que recibieron los pacientes terminada la endodoncia, encontrándose que el $52.4 \%$ de ellos estaba rehabilitado mediante resina compuesta.

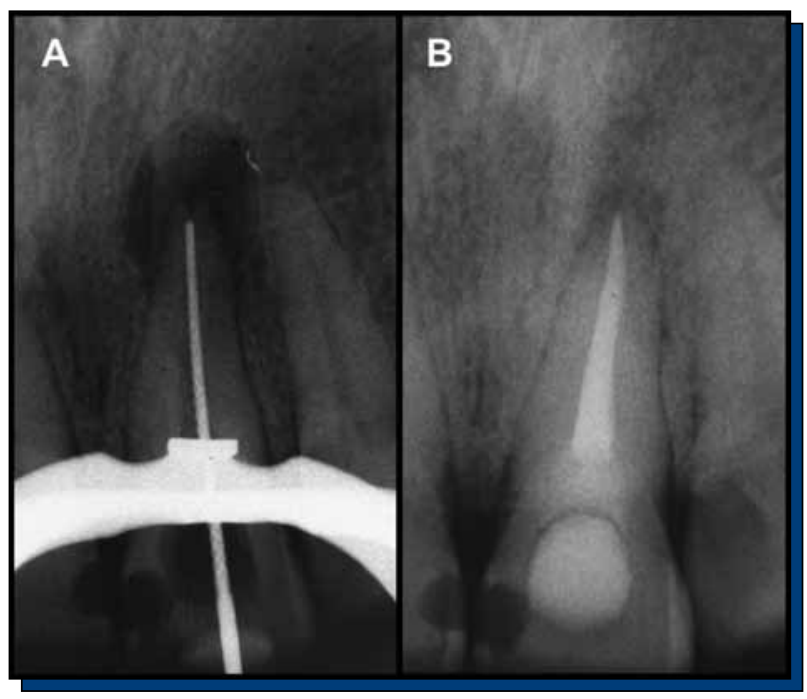

Figura 1. Ejemplo de tratamiento endodóntico definido como exitoso. A) Conductometría del diente 2.1 con una lesión periapical radiográfica evidente. B) Control a los 12 meses post-tratamiento, donde se observa reparación del tejido óseo.

Tabla 1. Frecuencia de pacientes según los distintos tipos de periodontitis apical.

\begin{tabular}{|c|c|c|}
\hline Patologías & $\mathbf{N}^{\circ}$ de Pacientes & $\%$ \\
\hline PAS & 5 & 2.0 \\
\hline AAA & 17 & 6.6 \\
\hline AAC & 23 & 9.0 \\
\hline PAA & 49 & 19.1 \\
\hline Otras & $\mathbf{1 6 2}$ & $\mathbf{6 3 . 3}$ \\
\hline Total PA & $\mathbf{9 4}$ & $\mathbf{3 6 . 7}$ \\
\hline Total & $\mathbf{2 5 6}$ & $\mathbf{1 0 0}$ \\
\hline
\end{tabular}

PAS: Periodontitis Apical Sintomática. AAA: Absceso Apical Agudo. AAC: Absceso Apical Crónico. PAA: Periodontitis Apical Asintomática. *Se contabilizan 94 pacientes debido a que dos de los 92 pacientes presentaron dos patologías distintas.

Tabla 2. Distribución de periodontitis apicales agudas y crónicas según dientes afectados.

\begin{tabular}{|l|c|c|c|c|c|}
\hline $\begin{array}{l}\text { Patologías } \\
\text { agudas }\end{array}$ & $\begin{array}{l}\mathbf{N}^{\circ} \\
\text { Dientes }\end{array}$ & $\%$ & $\begin{array}{l}\text { Patologías } \\
\text { crónicas }\end{array}$ & $\begin{array}{l}\mathbf{N}^{\circ} \\
\text { Dientes }\end{array}$ & $\%$ \\
\hline PAS & 5 & 5.1 & AAC & 23 & 23.2 \\
\hline AAA & 17 & 17.1 & PAA & 54 & 54.6 \\
\hline Total & $\mathbf{2 2}$ & $\mathbf{2 2 . 2}$ & Total & $\mathbf{7 7}$ & $\mathbf{7 7 . 8}$ \\
\hline
\end{tabular}


Tabla 3. Distribución de periodontitis apicales según edad y sexo de pacientes afectados.

\begin{tabular}{|l|c|c|c|c|c|c|}
\hline \hline Edades & Femenino & Masculino & Total & Femenino & Masculino & Total \\
\hline $10-19$ & 6 & 3 & 9 & $6.5 \%$ & $3.3 \%$ & $\mathbf{9 . 8 \%}$ \\
\hline $20-29$ & 9 & 4 & 13 & $9.8 \%$ & $4.3 \%$ & $\mathbf{1 4 . 1 \%}$ \\
\hline $30-39$ & 13 & 4 & 17 & $14.1 \%$ & $4.3 \%$ & $\mathbf{1 8 . 4 \%}$ \\
\hline $40-49$ & 25 & 7 & 32 & $27.2 \%$ & $7.6 \%$ & $\mathbf{3 4 . 8 \%}$ \\
\hline $50-59$ & 11 & 1 & 12 & $12 \%$ & $1.1 \%$ & $\mathbf{1 3 . 1 \%}$ \\
\hline$\geq 60$ & 4 & 4 & 8 & $4.3 \%$ & $4.3 \%$ & $\mathbf{8 . 6 \%}$ \\
\hline$*$ & 1 & - & 1 & $1.1 \%$ & - & $\mathbf{1 . 1 \%}$ \\
\hline Total & $\mathbf{6 9}$ & $\mathbf{2 3}$ & $\mathbf{9 2}$ & $\mathbf{7 5 \%}$ & $\mathbf{2 5 \%}$ & $\mathbf{1 0 0 \%}$ \\
\hline
\end{tabular}

En cuanto al sexo, Jiménez et al. ${ }^{(8)}$, han indicado en su estudio una clara predominancia del sexo femenino, con un porcentaje $66 \%$, tendencia que no cambia en el nuestro, que presenta una frecuencia de un $75 \%$, no existiendo diferencias entre ambas investigaciones. En contraste, Kierkevang et al. ${ }^{(18)}$ en su estudio indicaron una predominancia de periodontitis apical en el sexo masculino, con un porcentaje de un $52 \%$. Esta predominancia del sexo femenino en nuestro estudio y en el de Jiménez et al.(8) podría ser explicada por una mayor preocupación y por tanto de consulta de éstas frente a sus problemas de salud, siendo por tanto mas registradas.

En relación a la edad, Gulsahi et al. y Kabak et al. ${ }^{(4,15)}$, muestran en sus estudios una mayor frecuencia de la $4^{\text {ta }}$ y $5^{\text {ta }}$ década, con porcentajes del $24 \%$ y del $22 \%$ respectivamente, lo que se asemeja al nuestro, que tiene en la $5^{\text {ta }}$ década su mayor frecuencia con un $34.8 \%$, sin embargo, la diferencia en nuestro trabajo es mayor. La mayor frecuencia en pacientes de la $5^{\text {ta }}$ década, y en general de los grupos etarios más jóvenes, podría deberse a que estos consultan más frente a los problemas dentales que se les presentan, además de poseer una mayor cantidad de dientes y por lo tanto mayor posibilidad de desarrollar la patología.

El éxito de tratamiento en dientes con patología periapical también ha sido ampliamente estudiado. Para esta variable nuestra investigación tuvo una muestra pequeña debido a que la mayoría de los pacientes citados no acudieron a control, lo que coincide con lo señalado en diversas publicaciones donde no más del $10 \%$ de los pacientes acuden al control a largo plazo ${ }^{(12,14)}$. De esta forma acudieron al control clínico radiográfico, a los 6 meses o más, 21 pacientes, verificándose el éxito del tratamiento en el $100 \%$ de los casos. Este valor es similar al de Molven et al., quienes determinaron en su trabajo un porcentaje de un $95 \%$ de éxito(12). Otros estudios, como los de Estrela et al., Marques et al. y Sidaravicius et al. ${ }^{(7,11,13)}$, presentaron porcentajes de éxito de tratamiento de entre $62 \%$ al $78 \%$, los que son menores a nuestro estudio. La diferencia con estos estudios probablemente sea explicada por el uso de distintos protocolos en la realización de los tratamientos endodónticos por parte de los profesionales pertenecientes a dichas poblaciones. La única paciente del presente estudio que señaló una leve molestia a la percusión, presentaba su obturación coronal en contacto prematuro y la radiografía indicaba regresión de la lesión periapical inicial, por lo que al eliminar el contacto prematuro y controlarla dos semanas después la molestia desapareció, confirmando el éxito del tratamiento endodóntico. Con respecto al tipo de rehabilitación de los dientes tratados endodónticamente, existió una predominancia de dientes restaurados con resina compuesta $(52.4 \%)$, y en segundo lugar con vidrio ionómero (5 de los 21 dientes examinados), cemento provisorio que por protocolo clínico debe ser colocado al finalizar el tratamiento antes de la derivación para su restauración definitiva. Este aspecto resulta interesante de considerar debido a que está ampliamente descrito que la rehabilitación posterior de un diente tratado endodónticamente constituye un factor clave para el éxito o fracaso del tratamiento en términos de reparación tisular ${ }^{(19,20)}$. De esta forma, desde un punto de vista docente resulta importante reforzar estos conocimientos en el estudiante ya que algunos de los pacientes relataron que no se les señaló la necesidad e importancia de la realización de una restauración definitiva.

Nuestros resultados nos permiten concluir que la frecuencia de periodontitis apical en pacientes tratados en la clínica de pregrado de endodoncia en la Facultad de Odontología de la Universidad de Chile en el año 2009 corresponde a un $36.7 \%$, siendo ésta menor que lo descrito en publicaciones realizadas en otras poblaciones. El éxito de tratamiento de los pacientes fue de un $100 \%$, sin embargo dado el bajo número de pacientes que concurrieron a la citación debe considerarse como un reporte preliminar.

\section{CONFLICTOS DE INTERÉS}

Los autores declaran no tener conflicto de interés alguno.
Endodoncia, de consultas de urgencia las cuales se caracterizan por una mayor cantidad de patologías agudas dentro de sus diagnósticos.

\section{REFERENCIAS BIBLIOGRÁFICAS}

1. Cohen S, Hargreaves K. Vías de la Pulpa. Editorial Elsevier España 9a edición, capítulo 14, 2007.

2. Lasala A. Endodoncia. Editorial Masson-Salvat $4^{\text {ta }}$ edición, capítulo 4, 1992.

3. Nair PNR. Pathogenesis of apical periodontitis and the causes of endodontic failures. Crit Rev Oral Biol Med, 2004; 15(6): 348-381. 4. Gulsahi K, Gulsahi A, Ungor M, Genc Y. Frequency of root-filled teeth and prevalence of apical periodontitis in an adult Turkish population. Int Endod J, 2008; 41: 78-85. 
5. Kawashima N, Wadachi R, Suda H, Yeng T, Parashos P. Root canal medicaments. Int Dent J, 2009; 59(1): 5-11.

6. Tervit C, Paquette L, Torneck C, Basrani B, Friedman S. Proportion of healed teeth with apical periodontitis medicated with two percent chlorhexidine gluconate liquid: A case-series study. J Endod, 2009; 35: 1182-1185.

7. Estrela C, Leles CR, Hollanda AC, Moura MS, Pécora J. Prevalence and risk factors of apical periodontitis in endodontically treated teeth in a selected population of Brazilian adults. Braz Dent J, 2008; 19(1): 34-39.

8. Jiménez A, Segura JJ, Poyato M, Velasco E, Ríos JV. Prevalence of apical periodontitis and frequency of root-filled teeth in an adult Spanish population. Int Endod J, 2004; 37: 167-173.

9. Valois C, Costa E. Periapical cyst repair after nonsurgical endodontic therapy - case report. Braz Dent J, 2005; 16(3): 254-258.

10. Walton R, Torabinejad M. Endodoncia: Principios y práctica. Editorial McGraw-Hill Interamericana, Capítulo 3, 1997.

11. Márques Md, Moreira B, Eriksen HM. Prevalence of apical periodontitis and results of endodontic treatment in an adult Portuguese population. Int Endod J, 1998; 31: 161-165.

12. Molven O, Halse A, Fristad I, MacDonald-Jankowski D. Periapical changes following root-canal treatment observed 20-27 years postoperatively. Int Endod J, 2002; 35: 784-790.

13. Sidaravicius B, Aleksejuniene J, Eriksen HM. Endodontic treatment and prevalence of apical periodontitis in an adult population of Vilnius, Lithuania. Endod Dent Traumatol, 1999; 15: 210-215.

14 Friedman S, Mor C. The success of endodontic therapy-healing and functionality. J Calif Dent Assoc, 2004; 32(6): 493-503.

15. Kabak Y, Abbott PV. Prevalence of apical periodontitis and the quality of endodontic treatment in an adult Belarusian population. Int Endod $\mathrm{J}$, 2005; 38: 238-245.

16. AAE Consensus Conference Recommended Diagnostic Terminology. J Endod, 2009; 35: 1634.

17. Gutmann JL, Baumgartner JC, Gluskin AH, Hartwell GR, Walton RE. Identify and define all diagnostic terms for periapical/periradicular health and disease states. J Endod, 2009; 35:1658-1674.

18. Kirkevang L, Hörsted-Bindslev P, Orstavik D, Wenzel A. Frequency and distribution of endodontically treated teeth and apical periodontitis in an urban Danish population. Int Endod J, 2001; 34: 198-205.

19. Trope M, Commentary. "Biological restoration": root canal and coronal reconstruction. J Esthet Restor Dent, 2010; 22(3): 178.

20. Iqbal MK, Johansson AA, Akeel RF, Bergenholtz A, Omar R. A retrospective analysis of factors associated with the periapical status of restored, endodontically treated teeth. Int J Prosthodont, 2003; 16(1): 3138. 